\title{
INVESTIGACIÓN
}

Recibido: 16/03/2021 --- Aceptado: 16/03/2021 --- Publicado: 24/05/2021

\section{¡PIDE MI VINO!: ANÁLISIS CON EYE TRACKING DEL ETIQUETADO DE BOTELLAS DE VINO EN UNA FERIA NACIONAL DEL VINO}

\section{Order my wine!: eye tracking analysis of wine labelling at a national wine fair}

(87) Marta Retamosa Ferreiro'. Universidad de Castilla-La Mancha. España. Marta.retamosa@uclm.es.

81 Miguel Ángel Gómez Borja. Universidad de Castilla-La Mancha. España. MiguelAngel.GBorja@uclm.es.

88 Ángel Millán Campos. Universidad de Castilla-La Mancha. España.

Angel.millan@uclm.es.

\section{Cómo citar el artículo:}

Retamosa Ferreiro, M., Gómez Borja, M. Á. y Millán Campos, Á. (2021). ¡Pide mi vino!: análisis con eye tracking del etiquetado de botellas de vino en una feria nacional del vino. Vivat Academia. Revista de Comunicación, 154, 213-226. http://doi.org/10.15178/va.2021.154.e1344

http://www.vivatacademia.net/index.php/vivat/article/view/1344

\section{RESUMEN}

En una sociedad saturada e hipercomunicada, un diseño eficaz es clave para el éxito de un producto. Las técnicas neurocientíficas al servicio de la publicidad y el marketing constituyen herramientas efectivas a la hora de predecir el comportamiento del consumidor. Teniendo en cuenta este contexto y la gran diversidad de vinos que se comercializan en el mercado español, se pretende analizar el etiquetado de una selección de marcas y envases de vino. El objetivo principal de la investigación fue demostrar a las bodegas participantes cuales eran las zonas de sus etiquetados en los envases que despertaban mayor interés o engagement con su público objetivo. El estudio emplea un Eye Tracker de sobremesa y se desarrolló en la feria nacional de vino (FENAVIN) celebrada en Ciudad Real (Castilla La-Mancha) en 2019. La muestra

\footnotetext{
${ }^{1}$ Marta Retamosa Ferreiro: Doctora en Economía por la Universidad de Castilla-La Mancha por su tesis sobre Branding universitario. Sus áreas de investigación son la gestión de marca, el comportamiento del consumidor y el Neuromarketing.
} 
Retamosa Ferreiro, M., Gómez Borja, M, Á., y Millán Campos, Á.

¡Pide mi vino!: análisis con eye tracking del etiquetado de botellas de vino en una feria nacional del vino

objeto de análisis está integrada por especialistas del sector asistentes a la feria. Los resultados demostraron que las zonas superiores y centrales del etiquetado despertaban mayor interés, identificando las áreas ciegas que no reciben atención por parte de los sujetos analizados. El estudio concluye que el grado de atractivo y originalidad en el diseño de las etiquetas son factores determinantes clave en los procesos de percepción y la selección de los consumidores.

PALABRAS CLAVE: Neuromarketing - Eye tracking - etiquetado - packaging - áreas de atención - seguimiento ocular - botellas de vino.

\section{ABSTRACT}

In a saturated and hyper-communicated society, effective design is key to the success of a product. Neuroscientific tools at the service of advertising and marketing are effective instruments for predicting consumer behaviour. Considering this context and the great diversity of wines sold in the spanish wine market, the aim is to analyse the labelling of a selection of wine brands and bottles. The main objective of the research was to demonstrate to the wineries involved which areas of their labels on the bottles aroused the greatest interest or engagement with their target audience. The study employed a desktop eye tracker and it was carried out at the national wine fair (FENAVIN) held in Ciudad Real (Castilla La-Mancha) in 2019. The sample under analysis is comprised of sector specialists attending the fair. Findings revealed that the upper and central areas of the labelling were of greater interest, identifying the blind areas that were not attended by the sample. The study concludes that the degree of attractiveness and originality in label design are key determinants in consumer perception and selection processes.

KEYWORDS: Neuromarketing - Eye tracking - Labelling - Packaging - Area of attention - eye monitoring- wine bottles.

\section{INTRODUCCIÓN}

El marketing en la industria alimentaria influye en los consumidores al informarles sobre la existencia de nuevas marcas y productos, creando en estos de forma inconsciente un deseo de adquirirlos para degustarlos y así formarse una opinión de dicho producto (Chandon \& Wansink, 2012). Así, según estos autores los anuncios de productos alimentarios pueden utilizar una serie de mecanismos para conseguir incrementar la demanda de más cantidad de sus productos. Dentro de estos mecanismos destacan la modificación del precio de los alimentos tanto a corto como a largo plazo, realizar comunicaciones de marketing siendo riguroso con respecto a la calidad y cantidad del producto en sí y señalando la disponibilidad del producto alimentario en el entorno, es decir si el consumidor puede encontrar fácilmente el producto en su alrededor. En este punto, el etiquetado puede conformar también uno de estos elementos que logren incrementar la demanda de un determinado producto. 
Retamosa Ferreiro, M., Gómez Borja, M, Á., y Millán Campos, Á.

¡Pide mi vino!: análisis con eye tracking del etiquetado de botellas de vino en una feria nacional del vino

Sin embargo, hay muchos estímulos que no están directamente relacionados con un producto que puede impulsar la decisión del consumidor, como el brillo (Milosavljevic et al., 2011), los colores y la forma del envase (Itti \& Koch, 2001; Mannan et al., 2009), y precio (Oliver, 1997; Peng \& Wang, 2006; Cheng et al., 2008). De hecho, varios estudios han aplicado un enfoque de neuromarketing para investigar cómo la respuesta subconsciente y emocional a esas características podría influir en las preferencias (Stasi et al., 2018). Es el caso de un estudio de eye tracking realizado por Ares et al. (2013) que incluyó 53 consumidores a los que se les pidió que calificaran su percepción de la autenticidad de tres alimentos diferentes (mayonesa, pan y yogur) y su voluntad de comprar cada producto. Los resultados mostraron que las tres áreas más observadas para juzgar la autenticidad de un producto eran principalmente la marca, seguida de la lista de ingredientes y la información nutricional.

Para justificar el empleo de la herramienta neurocientífica de eye tracker en el estudio del etiquetado de alimentos, O'Connel et al. (2011) sostienen que el estudio del seguimiento ocular es útil para diversos ámbitos como puede ser la elaboración de publicidad y su evaluación, pruebas de logotipos, paquetes de diseño, etc. Más concretamente, en la siguiente tabla (véase tabla 1) se destacan algunos de los aspectos fundamentales del estudio del seguimiento de los ojos dentro del campo del neuromarketing, así como sus utilidades, ventajas y limitaciones:

Tabla 1: Aspectos fundamentales de la técnica eye tracking.

\begin{tabular}{|c|c|}
\hline ¿QUÉ MIDE? & $\begin{array}{ll}\text { - } & \text { Fijación visual } \\
\text { - } & \text { Búsqueda } \\
\text { - } & \text { Patrones de movimiento ocular } \\
\text { - } & \text { Resolución espacial } \\
\text { - } & \text { Atenoción } \\
\text { - } & \text { Dilatación de la pupila }\end{array}$ \\
\hline ¿CUÁNDO SE USA? & $\begin{array}{ll}\text { - } & \text { Prueba de sitios web } \\
\text { - } & \text { Pruebas de reacción en tiendas } \\
\text { - } & \text { Prueba de diseño de envases } \\
\text { - } & \text { Prueba de anuncios } \\
\text { - } & \text { Prueba de impresiones y diseño de imágenes } \\
\text { - } & \text { Probar cómo el consumidor filtra la información } \\
\text { - } & \text { Determinar la jerarquía de las percepciones de } \\
\text { - } & \text { Pruebúmulos de diseño de estanterías } \\
\text { - } & \text { Pruebas de colocación de productos }\end{array}$ \\
\hline VENTAJAS & $\begin{array}{l}\text { - Proporciona información precisa sobre la } \\
\text { participación en el procesamiento de imágenes y } \\
\text { grado de excitación } \\
\text { - Equipos portátiles que se pueden llevar a cualquier } \\
\text { ubicación } \\
\text { - Método no invasivo }\end{array}$ \\
\hline
\end{tabular}


Retamosa Ferreiro, M., Gómez Borja, M, Á., y Millán Campos, Á.

¡Pide mi vino!: análisis con eye tracking del etiquetado de botellas de vino en una feria nacional del vino

\begin{tabular}{l|ll}
\hline \multirow{3}{*}{ INCONVENIENTES } & - & El equipo es costoso \\
& - & Fiabilidad limitada en ocasiones \\
& - & Los resultados pueden depender de las condiciones \\
& oculares de los participantes.
\end{tabular}

Fuente: Adaptado de O'Connel (2011)

Como se ha mencionado anteriormente, el diseño del envase tiene, entre sus funciones, llamar la atención (Creusen \& Schoormans, 2005). Así como un envase bien diseñado puede tener efectos positivos, un diseño erróneo, puede provocar el efecto contrario, lo que implica conocer mejor cómo debe ser el diseño y cuál va a ser su impacto en las decisiones del cliente (Clement, 2007). Para captar esa atención es necesario captar la atención visual, que se manifiesta en el comportamiento ocular, comportamiento que se realiza inconscientemente, siendo ahí donde entra el eye tracker como la herramienta más apropiada para medirlo (Bridger \& Noble, 2015).

La atención visual actúa como un proyector, es el llamado enfoque selectivo del ojo que provoca un camino sobre un estímulo, en este caso el producto (Hübner et al., 2010). Cuando entramos en establecimiento de alimentación estamos rodeados de estímulos como la música, los olores, los carteles, la publicidad, etc., diseñados para atraer y, con frecuencia competir por captar la atención visual del cliente hacia una categoría de productos. Así, cualquier entorno comercial puede resultar muy complejo visualmente por el gran número de estímulos cognitivos existentes. De esta manera, el eye tracker permite conocer y analizar datos objetivos que establecen un vínculo entre cognición, compra y contacto visual (Lewinski et al., 2014).

En este contexto, la mejora de la publicidad de los productos debe centrarse en los aspectos que influyen en la atención y el comportamiento del consumidor. Para ello, se emplea el eye tracking, dado que suministra información de la citada atención de la persona analizada durante la exposición al estímulo.

Así, el motivo de haber seleccionado el sector vinícola y el mundo del vino como producto alimentario a analizar se debe a que según cifras de la Federación Española del Vino (FEV, 2020), España cuenta con 969.000 hectáreas de viñedo (el 13\% del total mundial) con cerca de 150 variedades autóctonas de uva plantadas y somos el tercer productor mundial. La facturación total de las bodegas españolas asciende a 6.500 millones de euros al año y el conjunto del sector representa alrededor del 1\% del PIB. Además, la FEV afirma que somos el primer exportador mundial en volumen, con algo más de 21 millones de hectólitros (2019). Y los terceros mayores exportadores del mundo en valor, con cerca de 2.700 millones de euros exportados en 2019. En España somos responsables del $25 \%$ de la producción de vino en Europa.

Por todo ello, en esta investigación se optó por la aplicación del Neuromarketing ya que según Boricean (2009), Ale Smidts definió en 2020 el término neuromarketing como el estudio del mecanismo cerebral para entender el comportamiento del consumidor con la finalidad de mejorar las estrategias de marketing. Es por esto que, tras elegir la mejor 
Retamosa Ferreiro, M., Gómez Borja, M, Á., y Millán Campos, Á.

¡Pide mi vino!: análisis con eye tracking del etiquetado de botellas de vino en una feria nacional del vino

herramienta para la finalidad del estudio, se ha empleado la herramienta neurocientífica eye tracker con el objetivo de obtener información del procesamiento del etiquetado de los sujetos analizados de las botellas de vino seleccionadas por las bodegas participantes.

\section{OBJETIVOS}

Dada la gran existencia de vinos en el mercado español el reto de las marcas es llamar la atención de los consumidores. Esta investigación pretende analizar qué es lo que llama la atención del consumidor y qué pasa desapercibido en el diseño del etiquetado de la botella de vino analizado. Por tanto, el objetivo principal de la investigación fue analizar y mostrar los datos obtenidos a las bodegas participantes sobre las zonas de sus etiquetados de las botellas de vino que despertaban mayor interés o engagement con su público objetivo. Para alcanzar el citado objetivo se proponen los siguientes objetivos específicos:

- Diseño de la investigación de acuerdo al etiquetado proporcionado por la propia bodega como apropiado para conocer el interés suscitado.

- Análisis con mapa de calor de los resultados obtenidos en la recolección de datos con el dispositivo eye tracker.

\section{METODOLOGÍA}

\subsection{Instrumento de medida}

Para la realización del experimento se ha empleado un eye tracker de sobremesa de la marca Gaze Point. De esta manera, y tal y como señalan O'Connel et al. (2011) el seguimiento ocular es apropiado para el desarrollo y la valoración de la usabilidad en línea, de anuncios, para el diseño de envases y logotipos, la elaboración de micrositios o para el marketing en la tienda. En este experimento, el etiquetado de las botellas de vino.

\subsection{Diseño del experimento}

Se ha elaborado una presentación con nueve imágenes o momentos. La primera y la última imagen contienen la explicación al inicio y a la finalización de la prueba. Otras siete imágenes muestran el etiquetado en las botellas de vino propuestos por las bodegas. De esas siete imágenes, se mostró en dos ocasiones el etiquetado de la botella fuera de su envase, es decir, se enseñó la imagen completa sin incluir la botella. Todas ellas se presentaron en un monitor al que se conectó el dispositivo de rastreo ocular. Durante el visionado de las imágenes, estas irán apareciendo una a una ocupando toda la pantalla del ordenador y con un tiempo de permanencia para cada imagen de entre 5 y 0,5 segundos. Cuando el participante estuvo sentado y relajado se le realizó una calibración con el dispositivo para asegurar que dicho dispositivo registraba correctamente el movimiento ocular. Seguidamente, se les pidió su completa atención al monitor y la realización del menor número de movimientos durante el experimento con el fin evitar alteraciones en el registro. 
Retamosa Ferreiro, M., Gómez Borja, M, Á., y Millán Campos, Á.

¡Pide mi vino!: análisis con eye tracking del etiquetado de botellas de vino en una feria nacional del vino

A continuación, se muestra un esquema del diseño del experimento mostrado a los participantes (véase Tabla 2):

Tabla 2: Secuencia de imágenes y tiempo del experimento

\begin{tabular}{|c|c|}
\hline IMAGEN & TIEMPO \\
\hline $\begin{array}{l}\text { Imagen de inicio con } \\
\text { explicación }\end{array}$ & 5 segundos \\
\hline Punto de fijación & 0,5 segundos \\
\hline Variedad 1 & 5 segundos \\
\hline Punto de fijación & 0,5 segundos \\
\hline Variedad 2 & 5 segundos \\
\hline Punto de fijación & 0,5 segundos \\
\hline Variedad 3 & 5 segundos \\
\hline Punto de fijación & 0,5 segundos \\
\hline Diseño 1 en botella & 5 segundos \\
\hline Punto de fijación & 0,5 segundos \\
\hline Diseño 1 sin botella & 5 segundos \\
\hline Punto de fijación & 0,5 segundos \\
\hline Diseño 2 en botella & 5 segundos \\
\hline Punto de fijación & 0,5 segundos \\
\hline Diseño 2 sin botella & 5 segundos \\
\hline Imagen de fin & 3 segundos \\
\hline
\end{tabular}

Fuente: Elaboración propia

\subsection{Muestra}

La obtención de la muestra se realizó en el mes de mayo de 2019 en Ciudad Real debido a que allí tiene lugar la celebración de la Feria Nacional del Vino (FENAVIN). Es una feria que convoca a un gran número de productores y comercializadores de vino a nivel nacional e internacional. Según una noticia de CMMMedia (2019), en esa edición de FENAVIN se reunieron 2.000 bodegas, 18.000 compradores y tuvieron lugar 7.000 reuniones comerciales. Para obtener los elementos a analizar, se hizo un llamamiento a las bodegas participantes sobre su participación en el experimento de su etiquetado con la herramienta de eye tracker.

Finalmente, dos bodegas participantes expresaron su interés en ser analizadas. Por el contrario, se obtuvo una muestra de sujetos analizados de 58 personas que quisieron someterse libre y voluntariamente al experimento. Es un tamaño suficiente dado que en neurociencia se admite este tamaño al no ser probabilístico (Casado- Aranda et al., 
Retamosa Ferreiro, M., Gómez Borja, M, Á., y Millán Campos, Á.

¡Pide mi vino!: análisis con eye tracking del etiquetado de botellas de vino en una feria nacional del vino

2020). Además, los participantes fueron informados en todo momento de la finalidad del estudio, se siguió un código ético en el diseño del experimento y se mantuvo el anonimato de los sujetos en la recolección de los datos.

\subsection{Representación gráfica de los resultados: Heat Map}

Para el análisis de los resultados obtenidos con el eye tracker, se ha optado por una de las representaciones más comúnmente empleada que son los mapas de calor. Consiguen hacer una representación más gráfica a través de la termografía. Muestran las zonas más calientes (normalmente representadas por colores cálidos como amarillo, rojo, naranja...), que indican los lugares o foco de interés donde el sujeto ha fijado su vista un mayor número de veces, frente a las zonas frías representadas por colores de una gama más fría (azul, verde...), que son las partes que no reciban mucha atención por parte del sujeto analizado.

\section{RESULTADOS}

A continuación, se muestran los resultados de los mapas de calor obtenidos para cada una de las bodegas analizadas y el etiquetado propuesto para su medición neurocientífica con eye tracker.

\subsection{Etiquetado Bodega 1}

Del etiquetado de esta botella se observa que los consumidores analizados han fijado su mirada en la red trazada por la raíz. Además, el color rojo obtiene mayor atención que otros colores empleados. Seguidamente el mayor número de fijaciones es recibido por el nombre "Campos Reales" para continuar leyendo la variedad, la procedencia y la añada. A continuación, el consumidor fijó su mirada en la graduación alcohólica situada en el margen inferior derecho. Se puede concluir que el diseño de la etiqueta es apropiado al captar la atención mayoritariamente en su parte central. Destaca además la sección superior del corcho que recibe un número importante de fijaciones (véase Imagen 1). 
Retamosa Ferreiro, M., Gómez Borja, M, Á., y Millán Campos, Á.

¡Pide mi vino!: análisis con eye tracking del etiquetado de botellas de vino en una feria nacional del vino
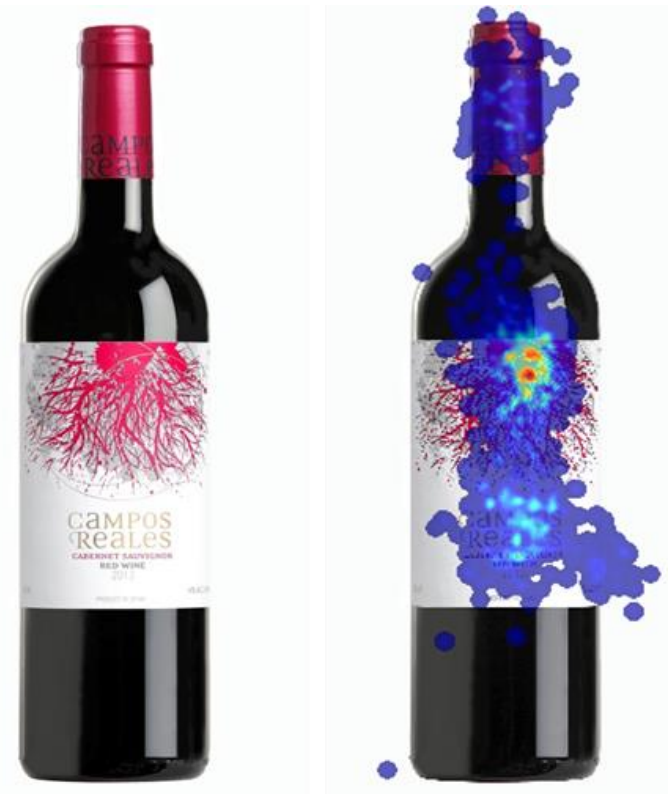

Imagen 1: Botella de vino Variedad 1 (antes y después del análisis con eye tracker). Fuente: Elaboración propia con programa Ogama (Open Gaze And Mouse Analyzer).

En el etiquetado de esta botella se observa que las miradas parten de la zona central y que poco a poco se disipan para abarcar el nombre "Campos Reales" y la descripción central de la etiqueta (variedad, procedencia y añada) como ocurría en el caso anterior. Sin embargo, el diseño de la izquierda recibe menos atención. Se puede deber a su posición en el margen izquierdo apartado de la zona central, además de su coloración oscura. Llama menos la atención, aunque recibe fijaciones puntuales por la singularidad del mismo. Al igual que se describía en el caso anterior, la graduación alcohólica situada en el margen inferior derecho recibe atención. La zona del corcho apenas recibe fijaciones por la coloración oscura (véase Imagen 2).

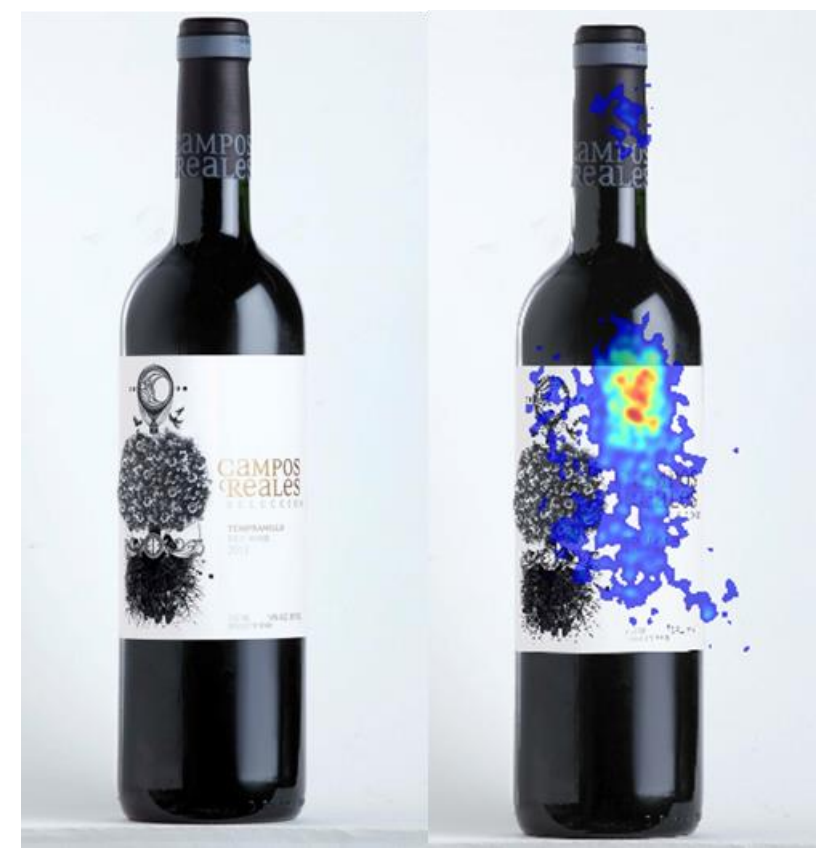


Retamosa Ferreiro, M., Gómez Borja, M, Á., y Millán Campos, Á.

¡Pide mi vino!: análisis con eye tracking del etiquetado de botellas de vino en una feria nacional del vino

Imagen 2: Botella de vino Variedad 2 (antes y después del análisis con eye tracker). Fuente: Elaboración propia con programa Ogama (Open Gaze And Mouse Analyzer).

$\mathrm{Al}$ igual que en el primer caso analizado, este etiquetado recibe un mayor número de fijaciones por la singularidad de su diseño y por encontrarse éste en la zona central de la botella. El toque de color amarillo/dorado de las aves capta la atención en contraste con el diseño oscuro. A continuación, las fijaciones se sitúan en el nombre "Campos Reales", en la variedad, procedencia, añada y posteriormente en la graduación. La zona del corcho recibe atención, aunque menor, por la coloración llamativa del mismo (véase Imagen 3).
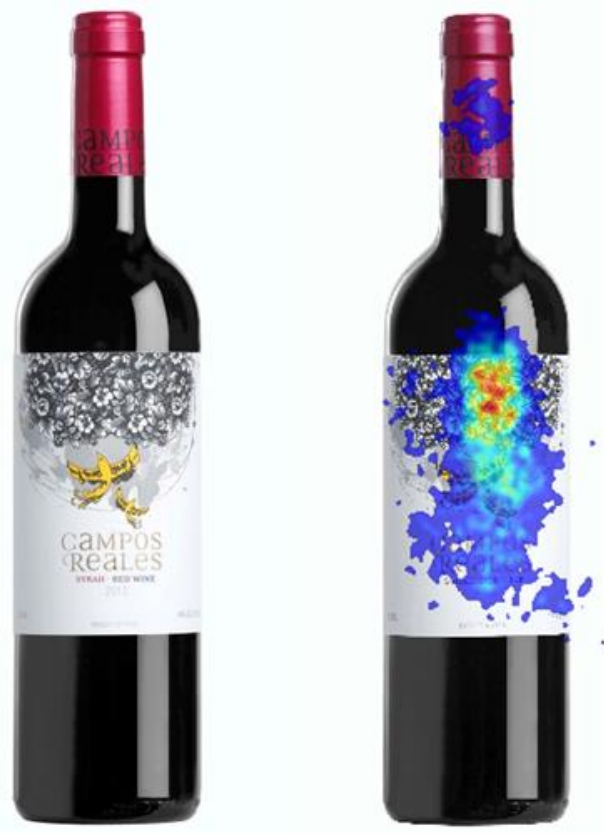

Imagen 3: Botella de vino Variedad 3 (antes y después del análisis con eye tracker). Fuente: Elaboración propia con programa Ogama (Open Gaze And Mouse Analyzer).

\subsection{Etiquetado Bodega 2}

Del etiquetado de esta botella, se pueden extraer conclusiones del diseño de forma aislada y este mismo etiquetado ya dispuesto en la botella. En la botella, se observa cómo las fijaciones de los consumidores se concentran en la cabeza de las aves entrelazadas. El color del diseño, rojo y oscuro, provoca un contraste atractivo. La parte superior de la campana, por la complejidad del diseño, reclama atención en su visionado. La parte superior con la descripción "Producto de España" recibe atención, además de la procedencia "Rioja" situada a continuación de la campana. La etiqueta que incluye el nombre "Zinio" no recibe fijaciones para este grupo de consumidores. Al analizar la etiqueta fuera del embotellado la fijación de las aves ahora se concentra en la parte superior de la campana. Se añade la atención captada por el etiquetado 
Retamosa Ferreiro, M., Gómez Borja, M, Á., y Millán Campos, Á.

¡Pide mi vino!: análisis con eye tracking del etiquetado de botellas de vino en una feria nacional del vino

inferior "Zinio" y la leyenda "Rioja" situada en la parte central del diseño (véase Imagen 4).
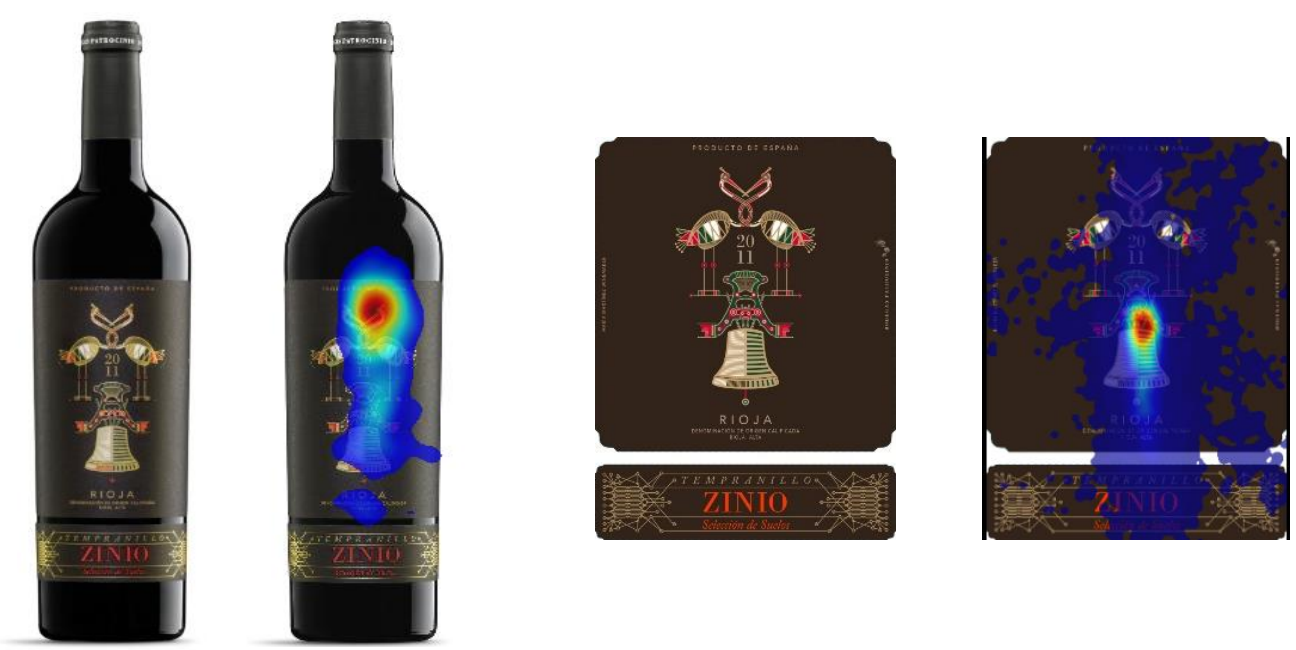

Imagen 4: Botella de vino Diseño 1 (antes y después del análisis con eye tracker) y etiquetado aislado (antes y después del análisis con eye tracker).

Fuente: Elaboración propia con programa Ogama (Open Gaze And Mouse Analyzer).

En este etiquetado, ocurre un efecto similar al anterior etiquetado analizado debido a que se trata de un diseño igual con la diferencia del fondo en color blanco. En la botella las fijaciones son recibidas por la palabra "Zinio" y la cabeza de las aves entrelazadas. La parte superior de la campana recibe atención y a continuación las fijaciones procederían a situarse en la procedencia "Rioja" situada en la parte inferior. En el caso de la etiqueta fuera del producto, vemos como la campana es la parte con mayor número de observaciones debido a la complejidad de su diseño, además de los colores empleados (rojo y verde oscuro). Además, la procedencia "Rioja" y "Zinio" destacan entre las fijaciones de los consumidores (véase Imagen 5). 
Retamosa Ferreiro, M., Gómez Borja, M, Á., y Millán Campos, Á.

¡Pide mi vino!: análisis con eye tracking del etiquetado de botellas de vino en una feria nacional del vino
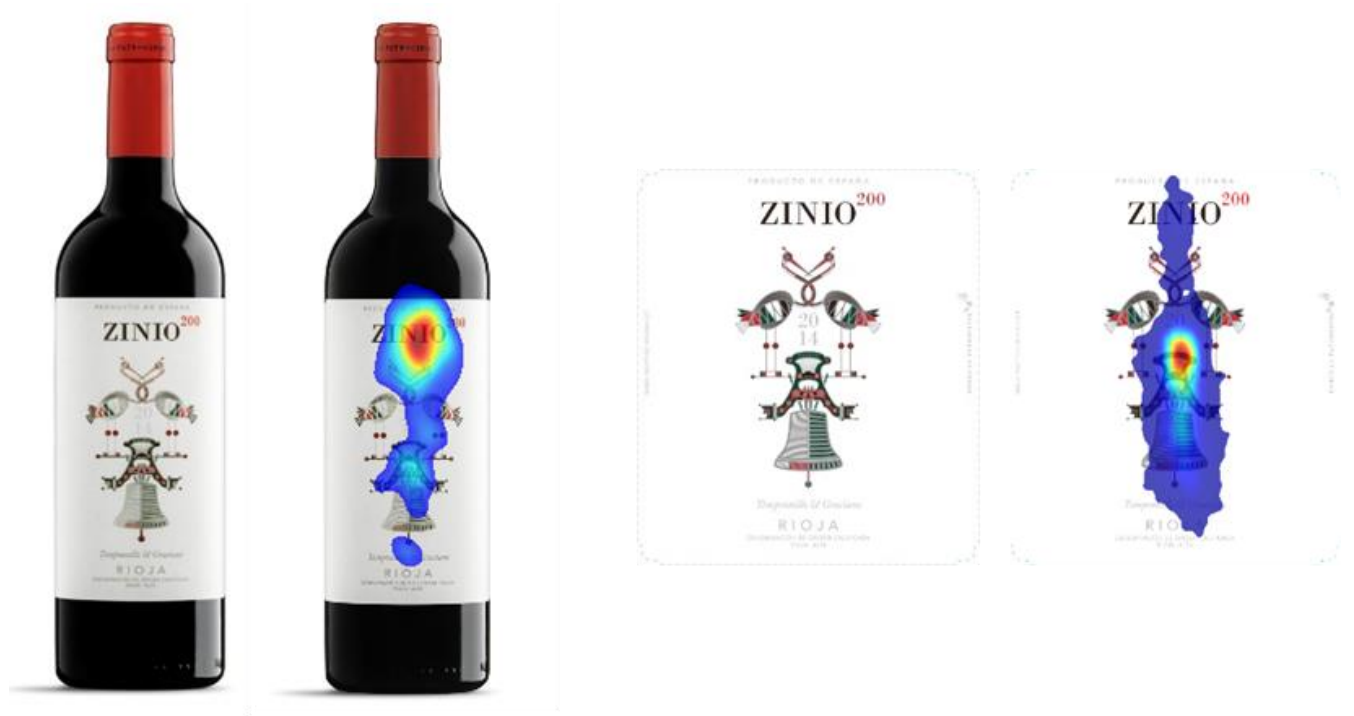

Imagen 5: Botella de vino Diseño 2 (antes y después del análisis con eye tracker) y etiquetado aislado (antes y después del análisis con eye tracker).

Fuente: Elaboración propia con programa Ogama (Open Gaze And Mouse Analyzer).

\section{DISCUSIÓN}

El sector vinícola en España presenta cifras que lo sitúan como uno de los más importantes de la economía. Por ello, el etiquetado de una botella de vino supone la comunicación de la marca al consumidor que va a disfrutar del producto. Es un atributo clave para transmitir lo que la empresa quiere sobre lo que está ofreciendo al mercado.

En cuanto a la idoneidad del uso en esta investigación de la herramienta de eye tracker, diversos autores como Laubrock et al. (2007) afirman que el seguimiento ocular permite medir el foco de atención. Así, como consideración general a los resultados, se observa que debido al patrón $\mathrm{Z}$ de lectura del ser humano (de izquierda a derecha y de arriba hacia abajo) la manera en qué un consumidor observa la etiqueta se realiza de arriba hacia abajo. Los elementos que quedan apartados de la línea vertical central obtienen menor atención, existiendo excepciones por diseños más atractivos o llamativos que logran superar este comportamiento.

Además, en esta investigación se ha comprobado que etiquetados con dibujos conceptuales o abstractos requieren una mayor atención del consumidor para descifrarlos, corriendo peligro la atención dedicada a otros elementos. Esta conclusión es lógica dado que en publicidad es muy relevante superar las barreras de la percepción y lograr que el mensaje sea asimilado y retenido por el receptor.

Como recomendación se propone a las bodegas cuidar sus diseños de los envases de las botellas ya que cuanto más acertado sea el diseño, más llamará la atención del consumidor. Y si se incrementa su atención, también aumentará su curiosidad e 
Retamosa Ferreiro, M., Gómez Borja, M, Á., y Millán Campos, Á.

¡Pide mi vino!: análisis con eye tracking del etiquetado de botellas de vino en una feria nacional del vino

intención de compra, logrando mayores posibilidades de venta del producto. Como consecuencia lógica la empresa podrá captar un mayor número de clientes (nuevos y usuales) $y$, por consiguiente, mayores ventas.

Además, el uso del eye tracker permitirá al empresario especificar aquellos factores clave para elaborar un diseño de envase en sintonía con los valores de la marca. Esta herramienta posibilitará también identificar cuáles son los elementos gráficos y estéticos clave del diseño.

Por tanto, dada la afirmación de diversos autores sobre que el neuromarketing aún está en desarrollo debido a su condición de disciplina joven, surge como futura línea de investigación la continuación en el aprendizaje sobre la mejora en la interpretación de los resultados obtenidos con herramientas neurocientíficas como el eye tracker en ese caso.

\section{REFERENCIAS}

Ares, G., Giménez, A. N. A., Bruzzone, F., Vidal, L., Antúnez, L., \& Maiche, A. (2013). Consumer visual processing of food labels: Results from an eye tracking study. Journal of Sensory Studies, 28(2), 138-153. https://doi.org/10.1111/joss.12031

Boricean, V. (2009, 14 - 15th, November). Brief history of Neuromarketing [ponencia]. ICEA - FAA, Bucharest.

Bridger, D., \& Noble, T. (2015). New tools and techniques for understanding nonconscious consumer decisions. Applied Marketing Analytics, 1(3), 214-220.

Casado-Aranda, L. A., Sánchez-Fernández, J., \& Ibáñez-Zapata, J. Á. (2020). Evaluating Communication Effectiveness through Eye Tracking: Benefits, State of the Art, and Unresolved Questions. International Journal of Business Communication, https://doi.org/10.1177/2329488419893746

Chandon, P. \& Wansink, B. (2012). Does food marketing need to make us fat? A review and solutions. Nutrition reviews, 70(10), 571-593.

Cheng, T. E., Lai, L. C., \& Yeung, A. C. (2008). The driving forces of customer loyalty: a study of internet service providers in Hong Kong. International Journal of EBusiness Research (IJEBR), 4(4), 26-42.

Clement, J. (2007). Visual influence on in-store buying decisions: an eye-track experiment on the visual influence of packaging design. Journal of marketing management, 23(9-10), 917-928.

CMMMedia (2019). FENAVIN en cifras. https://bit.ly/3a5Hgcr. 
Retamosa Ferreiro, M., Gómez Borja, M, Á., y Millán Campos, Á.

¡Pide mi vino!: análisis con eye tracking del etiquetado de botellas de vino en una feria nacional del vino

Creusen, M. E., \& Schoormans, J. P. (2005). The different roles of product appearance in consumer choice. Journal of product innovation management, 22(1), 63-81. https://doi.org/10.1111/j.0737-6782.2005.00103.x

Federación Española del Vino (FEV) (2020). Datos del sector vinícola español. http://www.fev.es/sector-cifras/

Hübner, R., Steinhauser, M., \& Lehle, C. (2010). A dual-stage two-phase model of $\begin{array}{lllll}\text { selective attention. } & \text { Psychological review, }\end{array}$ https://doi.org/10.1037/a0019471

Itti, L., \& Koch, C. (2001). Computational modelling of visual attention. Nature reviews neuroscience, 2(3), 194-203. https://doi.org/10.1038/35058500

Laubrock, J., Engbert, R., Rolfs, M. \& Kliegl, R. (2007). Microsaccades are an index of covert attention: Commentary on Horowitz, Fine, Fencsik, Yurgenson, Wolfe. Psychological Science, 18(4), 364-366. https://doi.org/10.1111/j.1467$\underline{9280.2007 .01904 . x}$

Lewinski, P., Fransen, M. L., \& Tan, E. S. (2014). Predicting advertising effectiveness by facial expressions in response to amusing persuasive stimuli. Journal of $\begin{array}{lllll}\text { Neuroscience, Psychology, and } & \text { Economics, }\end{array}$ https://doi.org/10.1037/npe0000012

Mannan, S. K., Kennard, C., \& Husain, M. (2009). The role of visual salience in directing eye movements in visual object agnosia. Current biology, 19(6), 247-248. https://doi.org/10.1016/j.cub.2009.02.020

Milosavljevic, M., Navalpakkam, V., Koch, C., \& Rangel, A. (2011). Relative visual saliency differences induce sizable bias in consumer choice. Journal of Consumer Psychology, 22(1), 67-74. https://doi.org/10.1016/j.jcps.2011.10.002

O'Connel, B., Walden, S. \& Pohlmann, A. (2011). Marketing and Neuroscience. What Drives Customer Decisions? American Marketing Association, White Paper.

Oliver, R. L. (1999). Whence consumer loyalty? Journal of marketing, 63(4_suppl1), 3344. https:// doi.org/10.1177/00222429990634s105

Peng, L. Y., \& Wang, Q. (2006). Impact of relationship marketing tactics (RMTs) on switchers and stayers in a competitive service industry. Journal of marketing management, 22(1-2), 25-59. https://doi.org/10.1362/026725706776022263

Stasi, A., Songa, G., Mauri, M., Ciceri, A., Diotallevi, F., Nardone, G., \& Russo, V. (2018). Neuromarketing empirical approaches and food choice: A systematic 
Retamosa Ferreiro, M., Gómez Borja, M, Á., y Millán Campos, Á.

¡Pide mi vino!: análisis con eye tracking del etiquetado de botellas de vino en una feria nacional del vino

review. Food

Research

International, 108,

650-664.

https://doi.org/10.1016/j.foodres.2017.11.049

\section{AUTOR/ES:}

\section{Marta Retamosa Ferreiro:}

La Dra. Marta es profesora del Departamento de Marketing de la Universidad de Castilla La Mancha. Marta ha obtenido su doctorado en economía reconocido internacionalmente con una tesis sobre la marca universitaria. Posee títulos de especialista en marketing internacional (IEB), máster universitario en estrategia y marketing de la empresa (UCLM) y máster universitario en Neuromarketing (UNIR). Sus campos de investigación son el branding, la gestión de marcas, el comportamiento del consumidor y el Neuromarketing, participando en numerosas charlas de divulgación sobre ello.

Orcid ID: https:/ / orcid.org/0000-0001-9984-7639.

Google Académico:

https://scholar.google.com/citations?user=T2ZzIPMAAAAJ\&hl=es

\section{Miguel Ángel Gómez Borja:}

El Dr. Miguel-Ángel Gómez-Borja es licenciado en Ciencias Económicas y Empresariales por la Universidad de Valencia y doctor en Administración de Empresas por la Universidad de Castilla-La Mancha. En la actualidad, es Catedrático de Marketing en la Facultad de Economía y Empresa de Albacete, España. Su investigación se centra, entre otros, en el impacto de las nuevas tecnologías de la información en la gestión de estrategias y tácticas de marketing, el comportamiento del consumidor en entornos virtuales y las herramientas y aplicaciones de investigación de marketing online, incluyendo el análisis de la neurociencia del consumidor. En cuanto a las aplicaciones sectoriales, sus intereses tienen mucho que ver con la agroalimentación, el turismo y el ocio, el comercio minorista y las nuevas tecnologías. También trabaja en temas relacionados con las organizaciones sin ánimo de lucro, la ayuda al desarrollo y los programas y herramientas de desarrollo sostenible.

Orcid ID: https:// orcid.org/0000-0002-9788-8629.

Google Académico:

https://scholar.google.es/citations?user=tJGAc60AAAAJ\&hl=es\&oi=sra

\section{Ángel Millán Campos:}

El Dr. Ángel Millán es profesor titular del Departamento de Marketing de la Universidad de Castilla La Mancha. Es autor de varios artículos académicos y libros sobre turismo, comportamiento del consumidor y branding. Ha publicado en revistas internacionales como Psychology \& Marketing y Journal of Brand Management.

Orcid ID: https:// orcid.org/0000-0002-9362-1513.

\section{Google Académico:}

https://scholar.google.es/citations?user=xBdhaZwAAAAJ\&hl=es\&oi=sra 